\title{
Adding exercise or subtracting sitting time for glycaemic control: where do we stand?
}

\author{
Paddy C. Dempsey ${ }^{1,2} \cdot$ Megan S. Grace ${ }^{1,2,3}$ - David W. Dunstan ${ }^{1,2,4,5,6}$
}

Received: 18 November 2016/Accepted: 29 November 2016/Published online: 12 December 2016

(C) Springer-Verlag Berlin Heidelberg 2016

\begin{abstract}
While regular structured exercise is a wellestablished (though arguably under-utilised) cornerstone in the prevention and management of type 2 diabetes, population adherence to recommended exercise guidelines remains stubbornly low. Indeed, most adults are exposed to environmental settings (at work, in automobile travel and in the domestic environment) that may not only limit their physical activity, but also promote sitting for prolonged periods of time. However, recent experimental evidence indicates that reducing and breaking up sitting time may also be a useful strategy to improve glycaemic control. In this issue of Diabetologia, Duvivier and colleagues report findings which suggest that reducing sitting time with standing and light-intensity activity could be a potential alternative to structured exercise for improving glycaemic control in type 2 diabetes patients. We review and discuss the findings of this study, its potential clinical implications, and a number of knowledge gaps and
\end{abstract}

Paddy C. Dempsey

paddy.dempsey@bakeridi.edu.au

1 Baker IDI Heart and Diabetes Institute, Level 4, 99 Commercial Rd, Melbourne, VIC 3004, Australia

2 Faculty of Medicine, Nursing and Health Sciences, Monash University, Melbourne, VIC, Australia

3 School of Health and Biomedical Sciences, RMIT University, Bundoora, VIC, Australia

4 Institute of Physical Activity and Nutrition Research, School of Exercise and Nutrition Sciences, Deakin University, Melbourne, VIC, Australia

5 School of Sport Science, Exercise and Health, The University of Western Australia, Perth, WA, Australia

6 Mary MacKillop Institute for Health Research, Australian Catholic University, Melbourne, VIC, Australia opportunities that could be considered in the interest of future research. The findings from Duvivier and colleagues should encourage healthcare practitioners, researchers and type 2 diabetes patients to consider the whole spectrum of physical activity, from sedentary behaviour through to structured exercise.

Keywords Cardiometabolic $\cdot$ Cardiovascular · Continuous glucose monitor $\cdot$ Diabetes $\cdot$ Energy expenditure $\cdot$ Exercise Glycaemic control $\cdot$ Lipids $\cdot$ Objective $\cdot$ Patterns $\cdot$ Physical activity $\cdot$ Public health $\cdot$ Sedentary $\cdot$ Sitting $\cdot$ Sitting

\section{Abbreviation \\ CGM Continuous glucose monitoring}

In our modern chair-dependant society, many people with type 2 diabetes may be sitting on a ticking time bomb. Recent epidemiological evidence, including a harmonised meta-analysis of 16 prospective studies with over 1 million individuals (2-18 years follow-up) [1] probably confirm what the Diabetologia readership already suspected - too much sitting and too little physical activity are bad news. Indeed, these studies indicate that low levels of physical activity and large volumes of sedentary time (sitting) are jointly associated with increased cardiometabolic morbidity and mortality in a dose-dependent manner [1-7]. However, for many of those with type 2 diabetes, the more pertinent question continues to linger: what are the most effective countermeasures? Should healthcare practitioners be preferentially encouraging 'good behaviours' (exercising more) or discouraging 'bad behaviours' (sitting less) to improve cardiometabolic health? Is there an 'optimal' approach?

In this issue of Diabetologia Duvivier and colleagues [8] report the results of an interesting experimental study in 19 physically inactive, overweight/obese adults with type 2 
diabetes. Using a randomised crossover design, the authors compared the effects of three 4 day activity regimens: (1) 'Sitting': 4415 steps/day with sitting 14 h/day; (2) 'Exercise': $4823 \mathrm{steps} /$ day with $1.1 \mathrm{~h} /$ day of sitting replaced with three consecutive $20 \mathrm{~min}$ bouts of moderate-to-vigorous cycling, performed at least $2 \mathrm{~h}$ after breakfast; and (3) 'Sit Less': 17,502 steps/day with $4.7 \mathrm{~h}$ /day of sitting replaced with intermittent standing $(2.5 \mathrm{~h})$ and light-intensity walking $(2.2 \mathrm{~h})$ across the day. Continuous glucose monitoring (CGM) was used to measure interstitial glucose levels throughout the activity regimens. Standardised meals were provided for dinner on day 3 and for all meals and snacks on day 4 of each regimen, and $24 \mathrm{~h}$ glucose control was assessed on day 4 using CGM. Fasting blood samples were collected on the morning of the fifth day for the assessment of glucose, insulin and lipids.

The study demonstrated that, compared with the Sitting condition, Sit Less reduced mean $24 \mathrm{~h}$ glucose levels, $24 \mathrm{~h}$ glucose excursions and duration of hyperglycaemia (glucose $>10 \mathrm{mmol} / \mathrm{l}$ ). Further, estimated insulin resistance (HOMA2IR) and fasting triacylglycerol levels were reduced on day 5 for Sit Less. While the Exercise protocol also tended to improve most of the above parameters, the magnitude of improvement for Exercise was less pronounced than for Sit Less, despite reportedly comparable energy expenditure.

\section{Why is this study of interest?}

Exercise is an established cornerstone in the management of type 2 diabetes. However, despite its known benefits, meeting prescribed exercise guidelines can be challenging and many with type 2 diabetes remain physically inactive [9-11]. Furthermore, sedentary behaviours increase the risk of type 2 diabetes and cardiovascular disease, even after controlling for leisure time spent in moderate-to-vigorous physical activity $[2,6]$. Therefore, reducing and breaking up prolonged sitting with more light-intensity activities may be a pragmatic option for type 2 diabetic individuals, particularly for those who are physically inactive, older, overweight/obese, or have reduced exercise tolerance [12].

The study by Duvivier and colleagues [8] is an important addition to the rapidly expanding evidence base on the glycaemic consequences of excessive sitting [7, 12-15]. However, only one previous experimental study has examined the effects of breaking up prolonged sitting with brief, intermittent bouts of light-intensity activities in a sample of type 2 diabetes patients, albeit under controlled experimental conditions [16]. Several aspects of the study by Duvivier and colleagues [8] are particularly noteworthy. These include:

- a comparison of the relative efficacy of structured Exercise vs Sit Less regimens against a prolonged Sitting condition
- approximate 'matching' of energy expenditure in the Sit Less and Exercise conditions using indirect calorimetry and objectively measured posture-sensitive activity monitors

- completion of each condition over consecutive days and under free-living conditions.

From a clinical perspective, the findings of this study have several potential implications for acute glycaemic control in adults with type 2 diabetes: (1) that morning bouts of structured exercise may not fully compensate for the negative glycaemic effects of prolonged sitting over the rest of the day, (2) that the duration of non-sitting activities may be more important than intensity for glycaemic control, or (3) that the higher frequency of interrupting sitting time (every $30 \mathrm{~min}$ ) compared with structured exercise, as well as its timing around meals, may have contributed to the more beneficial glycaemic effects.

\section{Study limitations and opportunities to develop the evidence base}

In the context of the present study, a number of opportunities could be considered in the interest of future research, including the following.

1. Feasibility and practicality As acknowledged by Duvivier and colleagues [8], the increased step count accomplished in the Sit Less condition (17,502 steps/day vs $\sim 4500$ steps/day on the Sitting condition) would generally be considered an 'extreme' prescription for most people of this age and/ or with pre-existing disease. Similarly, the intensity and duration of the Exercise condition (5.9 METs for $65 \mathrm{~min} /$ day) is well above current exercise recommendations. Thus, it seems unrealistic for a large proportion of this population group to achieve and sustain either activity regime in real-world settings. Importantly, whether greater improvements in glycaemic control would still be observed for the Sit Less compared with the Exercise conditions at more realistic activity volumes is uncertain, and warrants further investigation.

2. Energy expenditure Matching of energy expenditure and the achievement of 'energy balance' can be notoriously challenging in physical activity interventions. Indeed, the novelty and premise of the present study was predicated on the assumption that the Sit Less and Exercise conditions were roughly equivalent in terms of energy expenditure, which was achieved mainly through the inclinometer-estimated energy expenditure. However, it should be noted that all activity monitors inevitably have limited validity in determining energy expenditure across the activity spectrum, as this can only be inferred, not measured. Moreover, energy expenditure is 
driven by more than just bodily movement. Thus, it could be debated whether determining and matching energy expenditure is a realistic and ideal philosophy in studies that aim to reduce and interrupt sitting time- - when the assessment of relevant behaviours (e.g. sitting, standing and stepping of various intensities) and their specific contexts may be of more relevance [17]. With this in mind, the integration of data from multiple devices that are able to accurately capture context, posture and activity patterning/intensity in real-time could be emphasised in future research.

3. Activity dose, timing and patterning In the context of high levels of sitting across the population, identifying the doses, timing and patterning of physical activity that are required to optimise cardiometabolic health is of great clinical and practical relevance to type 2 diabetes management. In the present study [8], structured Exercise was completed $2 \mathrm{~h}$ after the breakfast meal, which may have afforded the Sit Less condition an unfair advantage. In other words, had the structured exercise bout been placed 30-60 min after breakfast, just prior to the breakfast meal, or in the evening, then the differences between the Sit Less and Exercise conditions for $24 \mathrm{~h}$ glucose control or fasting insulin/lipid measures may have been different. Further, it remains unknown whether those who exercise for 30-60 min in the morning or evening, but also reduce and break up their sitting time with more light-activity across the day, obtain additional glycaemic benefit compared with those exercising only (also see point 6 ).

4. Generalisability It will be important to establish the efficacy of interventions that reduce and break up sitting time in individuals with more advanced type 2 diabetes or with type 1 diabetes (i.e. those taking insulin). This is because of the increased likelihood of disturbances in glycaemic control and the risk of hypoglycaemia [18], along with potentially reduced responsiveness to exercisemediated glucose reductions [19]. It was encouraging that Duvivier and colleagues [8] observed more stable glucose levels during the Sit Less regimen compared with structured Exercise (assessed via 30 min glucose drops $>5 \mathrm{mmol} / \mathrm{l})$. However, in future studies it will be clinically relevant to examine additional metrics of glycaemic variability (see point 5 ) across a range of diabetic severities.

5. The untapped potential of CGM technologies The advent of CGM has made it possible to measure a continuous temporal line of interstitial glucose concentrations every 1$5 \mathrm{~min}, 24 \mathrm{~h} /$ day. This rich stream of data enables researchers to better characterise and understand both diurnal and nocturnal glycaemic responses in the context of their direction, periodicity and amplitude (i.e. glycaemic variability). Timestamped data can also be scrutinised in relation to meals and physical activity interventions. Moreover, CGM is minimally invasive and can be used outside of the laboratory in freeliving environments, providing new insights in real-world settings. In this context, there is great potential for CGM data to be integrated with objectively measured physical activity and sedentary behaviour devices. This would enable researchers to better identify activity patterns, interactions and dose-response relationships between diet, physical activity and sedentary time in relation to a suite of glycaemic parameters. Researchers are yet to use CGM data to its full capacity. However, it should also be made clear that, while CGM offers many clinical and research advantages, it is an imperfect surrogate for venous blood glucose. Signal stability, physiological differences in interstitial glucose homeostasis, physiological/device lag-times and individual differences are all factors that require consideration when interpreting CGM data [20, 21]. Further, since CGM only measures glucose levels, it does not provide any estimation of insulin sensitivity or beta cell function — - key information to fully understanding glycaemic responses.

6. Mechanisms and other outcomes Further investigation of the relevant mechanisms associated with the differential effects on metabolic control observed between the Sit Less and Exercise conditions is warranted. For example, it would be pertinent to understand whether there are varied changes in insulin signalling and/or hepatic glucose production in response to these interventions, and the relative timeline of such changes. Moreover, it would be highly relevant to understand whether the Sit Less and Exercise conditions differentially influence other biological markers associated with diabetes and cardiovascular disease risk (e.g. inflammation, metabolic and endothelial/cardiac function, oxidative stress, plasma and lipoprotein lipidomes, cognitive function and platelet function). In a broader sense, it seems likely that certain combinations of the Sit Less and Exercise regimens could be pursued to optimise both $24 \mathrm{~h}$ glycaemic control (via regular contraction-mediated glucose uptake) and cardiovascular function (increasing or maintaining endothelial function and cardiorespiratory fitness) respectively. Investigation and integration of such mechanisms and outcomes will be important in providing an informed basis for future interventions and clinical guidelines.

\section{Broadening the 'targeted' physical activity options for type 2 diabetes management}

The findings by Duvivier and colleagues [8] are timely in light of the American Diabetes Association's recently published position statement on exercise in type 2 diabetes, which now includes specific recommendations to reduce and interrupt prolonged sitting as part of a 'whole- day' approach to encourage more movement [22]. These recommendations, in 
addition to the promotion of moderate-to-vigorous exercise, corroborate growing concerns about the potentially deleterious role of prolonged and total daily sitting time in type 2 diabetes management. However, what is also clear is that the evidence to underpin public health and clinical initiatives with regard to sedentary behaviour reductions is not extensive. Most notably, generalisability to more realistic and freeliving settings, and the longer term clinical efficacy, feasibility and durability of reducing and breaking up prolonged sitting time in the management of type 2 diabetes remains largely unknown. Nevertheless, the increasing awareness of these concerns (i.e. total and prolonged sitting) and the potential for effective countermeasures (i.e. reducing and regularly interrupting prolonged sitting) are an important foundation upon which to build an evidence base to help inform future targeted longer term interventions, policies and programme development.

\section{Conclusion}

There are copious reasons to continue to emphasise the importance of regular structured moderate-to-vigorous exercise as a mainstay in type 2 diabetes management. However, with stubbornly low adherence to structured exercise programmes and an increasing shift in the balance of non-exercise (or background) activities towards prolonged sitting time, it seems prudent to also advise: 'Moving More AND Sitting Less' as part of a more malleable and comprehensive 'whole-day' approach. Indeed, this behavioural strategy may be an acceptable starting point (or stepping stone) for a large proportion of type 2 diabetes patients who are currently sedentary, deconditioned, or unable/reluctant to embark upon structured exercise. Moreover, environmental or occupational policies aiming to replace prolonged sitting with more light activity, particularly after meals, may prove particularly salient for improving glycaemic control. We commend Duvivier and colleagues [8] for their recent research efforts, which provide important insights and will help advance the field. Further experimental and longer-term intervention studies are now required to inform specific sedentary behaviour recommendations for clinical practice, and public health policies aiming to maximise cardiometabolic health benefits.

Funding All authors are supported by a NHMRC Centre of Research Excellence grant \#1057608 and the Victorian Government's Operational Infrastructure Support Program. DWD is supported by a NHMRC Senior Research Fellowship \#1078360.

Duality of interest statement The authors declare that there is no duality of interest associated with this manuscript.
Contributions statement PCD, MSG and DWD were all responsible for drafting the article, revising it critically for important intellectual content, and approving the final version for publication.

\section{References}

1. Ekelund U, Steene-Johannessen J, Brown WJ et al (2016) Does physical activity attenuate, or even eliminate, the detrimental association of sitting time with mortality? A harmonised meta-analysis of data from more than 1 million men and women. Lancet 388: $1302-1310$

2. Biswas A, Oh PI, Faulkner GE et al (2015) Sedentary time and its association with risk for disease incidence, mortality, and hospitalization in adults: a systematic review and meta-analysis. Ann Intern Med 162:123-132

3. Chau JY, Grunseit AC, Chey T et al (2013) Daily sitting time and all-cause mortality: a meta-analysis. PLoS One 8:e80000

4. Hu FB, Leitzmann MF, Stampfer MJ, Colditz GA, Willett WC, Rimm EB (2001) Physical activity and television watching in relation to risk for type 2 diabetes mellitus in men. Arch Intern Med 161:1542-1548

5. Hu FB, Li TY, Colditz GA, Willett WC, Manson JE (2003) Television watching and other sedentary behaviors in relation to risk of obesity and type 2 diabetes mellitus in women. JAMA 289:1785-1791

6. Wilmot EG, Edwardson CL, Achana FA et al (2012) Sedentary time in adults and the association with diabetes, cardiovascular disease and death: systematic review and meta-analysis. Diabetologia 55: 2895-2905

7. Brocklebank LA, Falconer CL, Page AS, Perry R, Cooper AR (2015) Accelerometer-measured sedentary time and cardiometabolic biomarkers: a systematic review. Prev Med 76:92-102

8. Duvivier BMFM, Schaper NC, Hesselink MKC et al (2016) Breaking sitting with light activities vs structured exercise: a randomised crossover study demonstrating benefits for glycaemic control and insulin sensitivity in type 2 diabetes. Diabetologia, doi:10.1007/s00125-016-4161-7

9. Brawner CA, Churilla JR, Keteyian SJ (2016) Prevalence of physical activity is lower among individuals with chronic disease. Med Sci Sports Exerc 48:1062-1067

10. Zhao G, Ford ES, Li C, Mokdad AH (2008) Compliance with physical activity recommendations in US adults with diabetes. Diabet Med 25:221-227

11. Cichosz SL, Fleischer J, Hoeyem P et al (2013) Objective measurements of activity patterns in people with newly diagnosed type 2 diabetes demonstrate a sedentary lifestyle. Diabet Med 30:10631066

12. Dempsey PC, Owen N, Yates TE, Kingwell BA, Dunstan DW (2016) Sitting less and moving more: improved glycaemic control for type 2 diabetes prevention and management. Curr Diab Rep 16: 114

13. Benatti FB, Ried-Larsen M (2015) The effects of breaking up prolonged sitting time: a review of experimental studies. Med Sci Sports Exerc 47:2053-2061

14. Chastin SF, Egerton T, Leask C, Stamatakis E (2015) Meta-analysis of the relationship between breaks in sedentary behavior and cardiometabolic health. Obesity (Silver Spring) 23:1800-1810

15. van der Berg JD, Stehouwer CD, Bosma H et al (2016) Associations of total amount and patterns of sedentary behaviour with type 2 diabetes and the metabolic syndrome: The Maastricht Study. Diabetologia 59:709-718 
16. Dempsey PC, Larsen RN, Sethi P et al (2016) Benefits for type 2 diabetes of interrupting prolonged sitting with brief bouts of light walking or simple resistance activities. Diabetes Care 39: 964-972

17. Kelly P, Fitzsimons C, Baker G (2016) Should we reframe how we think about physical activity and sedentary behaviour measurement? Validity and reliability reconsidered. Int J Behav Nutr Phys Act 13:32

18. American Diabetes Association (2015) Standards of medical care in diabetes - 2015. Diabetes Care 38(Suppl1):S1-S2

19. Solomon TP, Malin SK, Karstoft K, Haus JM, Kirwan JP (2013) The influence of hyperglycemia on the therapeutic effect of exercise on glycemic control in patients with type 2 diabetes mellitus. JAMA Intern Med 173:1834-1836

20. Wagner J, Tennen H, Wolpert H (2012) Continuous glucose monitoring: a review for behavioral researchers. Psychosom Med 74: 356-365

21. Damiano ER, McKeon K, El-Khatib FH, Zheng H, Nathan DM, Russell SJ (2014) A comparative effectiveness analysis of three continuous glucose monitors: the navigator, G4 platinum, and enlite. J Diabetes Sci Technol 8:699-708

22. Colberg SR, Sigal RJ, Yardley JE et al (2016) Physical activity/ exercise and diabetes: a position statement of the american diabetes association. Diabetes Care 39:2065-2079 